\title{
HITRAN online: An online interface and the flexible representation of spectroscopic data in the HITRAN database
}

\author{
C. Hill ${ }^{\mathrm{a}, \mathrm{b}, *}$, I. E. Gordon ${ }^{\mathrm{a}}$, R. V. Kochanov ${ }^{\mathrm{a}, \mathrm{c}}$, L. Barrett ${ }^{\mathrm{a}, \mathrm{d}}$, J. S. \\ Wilzewski ${ }^{\mathrm{a}, \mathrm{e}}$, L. S. Rothman ${ }^{\mathrm{a}}$ \\ ${ }^{a}$ Harvard-Smithsonian Center for Astrophysics, 60 Garden Street Cambridge MA 02138, \\ USA \\ ${ }^{b}$ University College London, Gower Street, London WC1E 6BT, UK \\ ${ }^{c}$ Laboratory of Quantum Mechanics of Molecules and Radiative Processes, Tomsk State \\ University, 36 Lenina Avenue, 634050 Tomsk, Russia \\ ${ }^{d}$ Department of Computer Science, University of Massachusetts Boston, 100 Morrissey \\ Boulevard, Boston MA 02125, USA \\ ${ }^{e}$ Now at Ludwig-Maximilians-Universität München, Faculty of Physics, Schellingstr. 4, \\ 80799 Munich, Germany and German Aerospace Center (DLR) Oberpfaffenhofen, 82234 \\ Weßling, Germany
}

\begin{abstract}
We describe a new, online interface to the HITRAN database that overcomes the many limitations of the existing, 160-character fixed-width, text based format (the ".par" files mostly distributed through an FTP site until now.) The interface, called HITRAN online, accesses a relational database [Hill et al. (2013)] in which the spectroscopic data are stored in a flexible, extensible and structured format. This allows an arbitrary number of different parameters for each transition to be stored so that HITRAN can represent, for example, non-Voigt line shape profiles as well as parameters representing broadening by species other than "air" and "self".

The online interface provides many ways of visualizing data as part of querying the database and allows users to create and save their own output formats to suit their own needs. A bibliography file produced with each data file provides citations and notes to the original data sources to make it easier for users to credit data providers. Once registered with the HITRANonline
\end{abstract}

\footnotetext{
${ }^{*}$ Corresponding author.
}

Preprint submitted to Journal of Quantitative Spectroscopy and Radiative TransferDecember 14, 2015

(C) 2015. This manuscript version is made available under the Elsevier user license http://www.elsevier.com/open-access/userlicense/1.0/ 
service, users also have (private) access to thier own search history which summarizes and can repeat queries.

Keywords: Line-lists, Radiative transfer, Databases, HITRAN

\section{Introduction}

The HITRAN database is a compilation of spectroscopic parameters used to simulate and analyse the transmission and emission of light in gaseous media, especially the terrestrial atmosphere. Since its inception in the late 1960s (when only the seven most infrared-active absorbers in the natural terrestrial atmosphere were included) it has undergone many revisions and extensions. At its latest major release ("HITRAN2012") [Rothman et al. (2013)] the line-by-line part of the database consisted of almost 4.5 million transitions for a total of 116 individual isotopologues of 47 different molecules. For each of these transitions, parameters are supplied to calculate its intensity and line shape as a function of temperature and pressure assuming a Voigt profile with a Lorentzian component determined by collisions with molecules of two types: "air" and "self".

The current format for the line-by-line part of the HITRAN database was last extended in the 2004 release [Rothman et al. (2005)] which expanded the text-based format to 160-characters per record allowing for reference and uncertainty indexes to be specified for six parameters, the inclusion of the Einstein $A$ coefficient and wider fields for the provision of quantum labels of the upper and lower states. A full list of parameters currently used in HITRAN (those provided in the 2004 format, referred to in this paper as the "native" HITRAN format (also called the par format after the extension used for these files), is given in Table 1. Where additional data were available for a molecule (such as lineshape parameters for non-Voigt profiles), these were provided in supplementary files on an ad hoc basis.

As the amount and quality of spectroscopic data has increased, particularly over the last 10 years, there is now a need for another format to store and present HITRAN data. The main reasons for this are summarized, with examples, below.

- With the recent interest in non-terrestrial atmospheres due to, for example, successful missions within the solar system such as Cassini [Jaffe and Herrell (1997)] and the increasing scope for measurements of the 
atmospheres of exoplanets [e.g. Charbonneau et al. (2002)], broadening by collisions with molecules other than "air" and "self" are important to HITRAN users. This entails the provision of a new set of $\gamma_{\mathrm{X}}, n_{\mathrm{X}}$, and perhaps $\delta_{\mathrm{X}}$ parameters (for a Voigt line shape) for each perturbing species, $\mathrm{X}$, where $\gamma_{\mathrm{X}}$ is the Lorentzian half-width at half-maximum, $n_{\mathrm{X}}$ is the temperature dependence exponent of this half-width, and $\delta_{\mathrm{X}}$ is the pressure-induced shift. Indeed, broadening parameters for several molecules pertubed by $\mathrm{H}_{2}$, He and $\mathrm{CO}_{2}$ have recently been introduced into the HITRAN compilation [Wilzewski et al. (2016)] (see Table 3).

- The increasing accuracy and resolution with which atmospheric absorption features can be measured has exposed the limitations of the Voigt profile as a model line shape for absorption (e.g. [Hartmann et al. (2008)] and references therein). More accurate line shapes taking into account a more sophisticated description of collisional processes, such as the Galatry [Galatry (1961)], Rautian ([Rautian and Sobel'Man (1966)]) and speed-dependent Voigt (e.g. [Boone et al. (2007)]) profiles require more parameters. A recent formulation of the partiallycorrelated quadratic speed-dependent hard-collision (pCqSDHC) line profile known as the Hartmann-Tran profile (HTP) [Ngo et al. (2013, 2014)] is parameterized in terms of six variables. There is no room in the current HITRAN fixed-length, text-based format for any further parameters to be specified.

- The native format allows for the isotopologue to be identified by only a single-digit integer. A few molecules have more than 10 significant isotopologues, and indeed data is available but not supplied in HITRAN2012 for the eleventh most-abundant isotopologue of carbon dioxide, ${ }^{18} \mathrm{O}^{13} \mathrm{C}^{17} \mathrm{O}$, and the tenth most-abundant isotopologue, ${ }^{13} \mathrm{C}^{18} \mathrm{O}_{2}$, was given the ID number 0 to fit it in the old format.

- Releasing data in a static format such as the .par text files comprising the HITRAN2012 line-by-line collection requires users to seek out updates periodically and download files from the HITRAN FTP server. Such data often replace older data which is then hard to find and makes reproducibility a challenge.

- References to the original sources of data in the HITRAN database have, until now, been provided as indexes (for each of the six parameters 
$\tilde{\nu}, S, \gamma_{\text {air }}, \gamma_{\text {self }}, n_{\text {air }}$ and $\left.\delta_{\text {air }}\right)$ in the .par format. The citation or note corresponding to this index is then looked up in a separate document, ref-table.pdf, provided and maintained on the HITRAN website. This procedure can be cumbersome for some users and consequently the original data providers do not always get the attribution they deserve in published work using HITRAN.

In this article we describe a new, online interface to the HITRAN database built on the relational database model described in [Hill et al. (2013)]. The relational database approach removes the constraints of fixed-width text fields for storage of parameters and allows an arbitrary number of parameters to be stored and retrieved for each transition, with their uncertainties and bibliographic references.

The parameter values are stored as double-precision floating point values (that is, to a maximum of about 15 significant digits of precision). All new data will be introduced into the database in this format and announcements will be placed on the HITRANonline news page as existing data is updated into this format. Note that for the case of line positions the HITRAN database was traditionally providing fixed width values with 6 figures after the decimal point, which did not provide sufficient accuracy in the microwave region. However, in HITRAN2012 the microwave line positions for $\mathrm{HNO}_{3}$ and $\mathrm{PH}_{3}$ (taken from JPL and CDMS databases respectively) were provided with floating point values and that is the way they can already be retrieved from HITRANonline.

The interface described in this article, HITRANonline, replaces and extends the JavaHAWKS application [Rothman et al. (1998)]. The online interface that was recommended with the HITRAN2012 edition was "HITRAN on the web" (http://hitran.iao.ru/), maintained at the Institute of Atmospheric Optics, Tomsk, Russia. However, "HITRAN on the web" does not presently represent extra parameters beyond those in HITRAN2012 and although it is updated regularly in close collaboration with the HITRAN group, it has a relatively unclear future. HITRANonline is the official conduit for HITRAN data and is maintained and overseen by the HITRAN group.

The HITRANonline service has been available at http://hitran.org since May 2015 and at the time of writing (December 2015) had over 1800 registered users. Registration, at http://hitran.org/register/ (and also linked from the home page), is free and requires the user to provide only a name and email address. 
The following section describes the process of querying and retrieving lineby-line data from this website, assuming the user is registered and logged in (http://hitran.org/login/). The third section describes the process of retrieving experimental cross sections. In the fourth section, the other resources available through HITRANonline, such as partition sums and the Python application programming interface library, HAPI, are summarized. The final section outlines planned expansions to the capabilities of the system.

\section{Line-by-line data}

\subsection{Searching for data}

Selection of transition data from HITRAN consists of several steps, followed in sequence to build a query of the database and to choose the output format of the data.

1. Select Molecules (http://hitran.org/lbl/). One or more molecules can be selected from a table listing all molecules for which HITRAN has line-by-line data (47 at the time of writing ${ }^{1}$ ). This table expands out to provide details of the number of lines, wavenumber range and intensity range for each molecule as well as a link to a concise overview spectrum to aid the selection of appropriate molecules. The concise overview is very useful in determining if HITRAN indeed has data within certain wavenumber ranges. The user can toggle between a linear and logarithmic scale, which is highly recommended in order to perceive weak bands in the database owing to the large dynamic range in intensities.

Figure 1 shows the top portion of the table for the first 11 molecules in HITRAN (the HITRAN numbering of molecules is somewhat arbitrary and is mainly the order in which they were added to the database). When Step 1 is completed, the next step button ("2. Select Isotopologes", above the table) is highlighted green to show it can be activated.

2. Select Isotopologues. For each of the molecules chosen in Step 1, details of that molecule's isotopologues are presented. Additional metadata

\footnotetext{
${ }^{1}$ Note, however, that for three of the molecules: $\mathrm{SF}_{6}$ (ID: 30), $\mathrm{ClONO}_{2}(35)$ and $\mathrm{CF}_{4}$ (42), line-by-line data is only available in the native .par format because these species have a very large number of lines.
} 
displayed include the isotopologues' natural (terrestrial) abundances, number of lines, wavenumber and intensity ranges. Figure 2 shows a menu for Step 2 after the user has chosen the molecule $\mathrm{HCl}$ in Step 1. This molecule has four isotopologues available in the database. When this step is completed, the next step button ("3. Select Wavenumber range") is highlighted green.

3. Select Wavenumber Range. This page allows users to optionally narrow down their search for lines to a particular wavenumber range (wavenumbers, that is, $\mathrm{cm}^{-1}$, are traditionally used for units in HITRAN). Since some molecules possess a great many lines, a judicious choice here can make the resulting data files more manageable. By default, all lines for all of the chosen isotopologues are requested. Figure 3 shows this feature.

4. Select or Create Output Format. Figure 4 illustrates this step. The default output format is the native, HITRAN2004 160-character .par format. This output format is always available to all users and clicking the green "5. Start Data Search" button will return data in this format. Of course, the parameters returned in this format are restricted to those of Table 1. Alternatively, a custom output format may be created and used as described below.

\subsection{Creating custom output formats}

The fourth step of searching the line-by-line portion of the HITRAN compilation contains an option to create a new output format (or to edit an existing one). The panel on the left hand side of this page lists available output formats (the .par format as well as any user-defined formats previously created and saved) and contains a button labelled "Create New Output Format". Clicking this opens up a page listing the selected parameters to output in the new format on the left and the available parameters (see Tables 1, 2 and 3) on the right. See Figure 5 for an illustration of this step: the sample output format depicted here shows some of the "traditional" parameters have been supplemented with line shape parameters describing broadening due to $\mathrm{CO}_{2}$. This output could be useful for scientists who study planets with $\mathrm{CO}_{2}$-dominated atmospheres as well as combustion. An output format is created by double-clicking (or dragging) parameters from the righthand panel. They can be re-ordered on the lefthand panel. Data for the most 


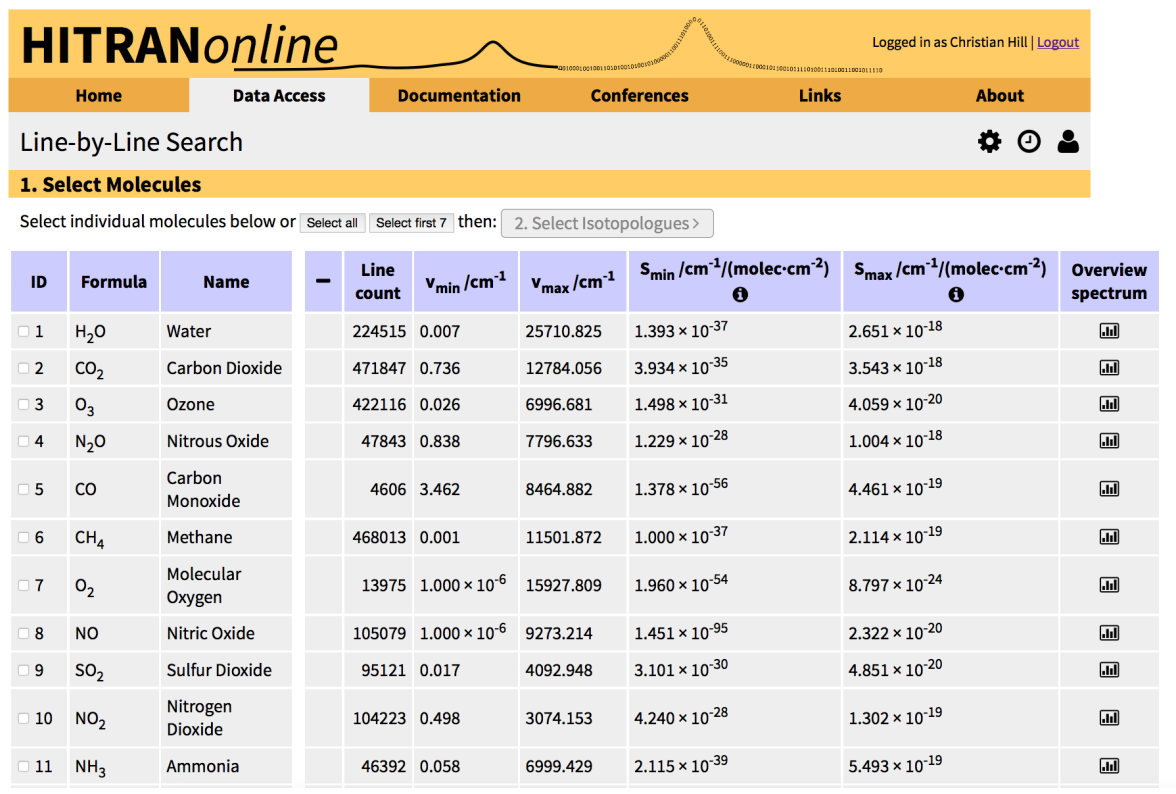

Figure 1: Step one for making a query of line-by-line data using the HITRANonline interface: Select Molecules.

important parameters in HITRAN are associated with references and uncertainties: these can be selected for inclusion in the output format by checking the corresponding columns.

Several of the parameters are special, "metadata" quantities such as identifying integers for the transitions, molecules and isotopologues. These are listed in Table 2 and, of course, do not have references or uncertainties associated with them. Users are warned if they try to create an output format which does not uniquely identify the species to which a transition belongs.

The "parameter" identified as ".par line" is representation of the transition in the native, 160-character HITRAN2004 format: appending additional parameters to an output format created with this choice as the first one is a convenient way to extend the existing format to include new data.

There are a few other options available for configuring a custom output format:

- the field separator (if any) can be set to allow, for example, commaseparated fields;

- HDF-5 output is supported; 


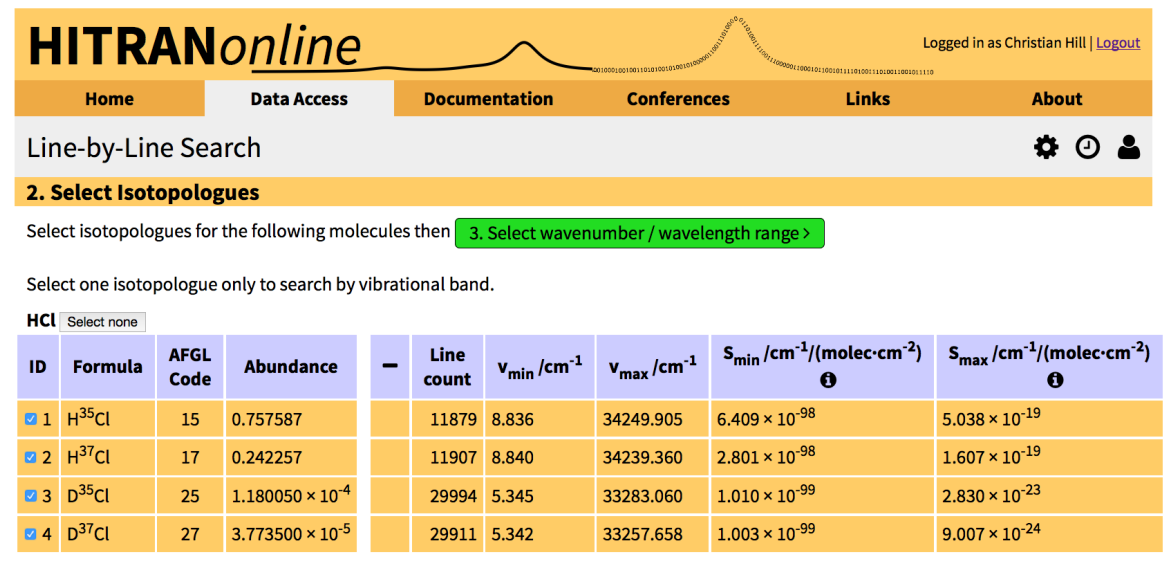

Figure 2: Step two for making a query of line-by-line data using the HITRANonline interface: Select Isotopologues.

- a header line identifying each column in the output can be selected.

Fixed-width or variable-width fields (columns) are possible: in the case of variable-width fields, a field separator must be chosen (even if it is just a single space). In the case of fixed-width fields, the format specifier of each parameter can be customized: this allows the number of characters in the field and the number of decimal places (for floating point quantities) to be chosen.

Each output format must be given a name and may also be given a more detailed description. On saving a newly-created or edited output format, the user is returned to the previous page and may start the data search by clicking the green "5. Start Data Search" button. 


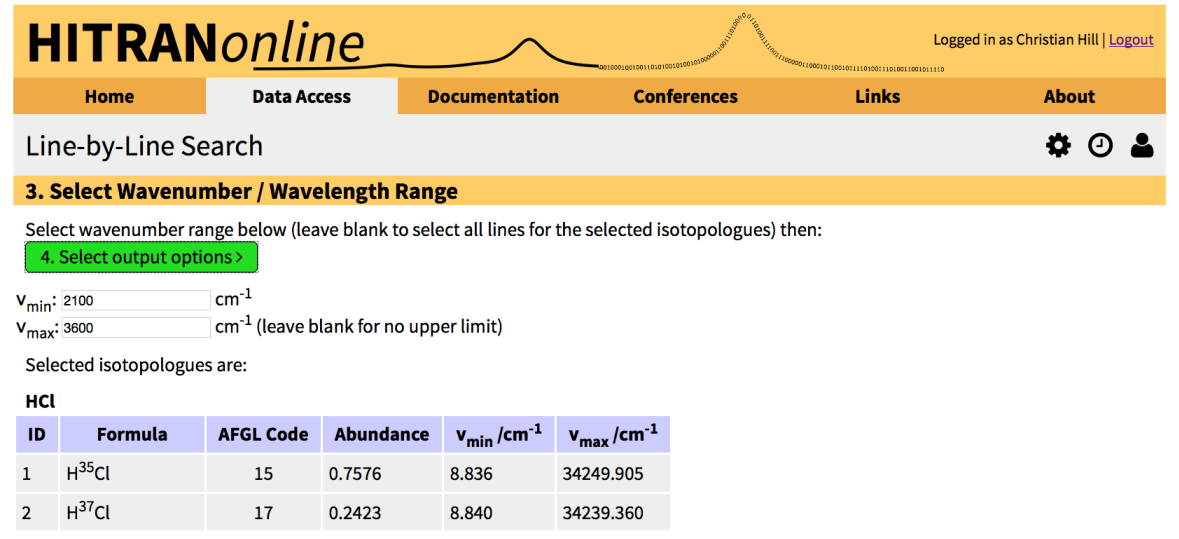

Figure 3: Step three for making a query of line-by-line data using the HITRANonline interface: Select Wavenumber Range.

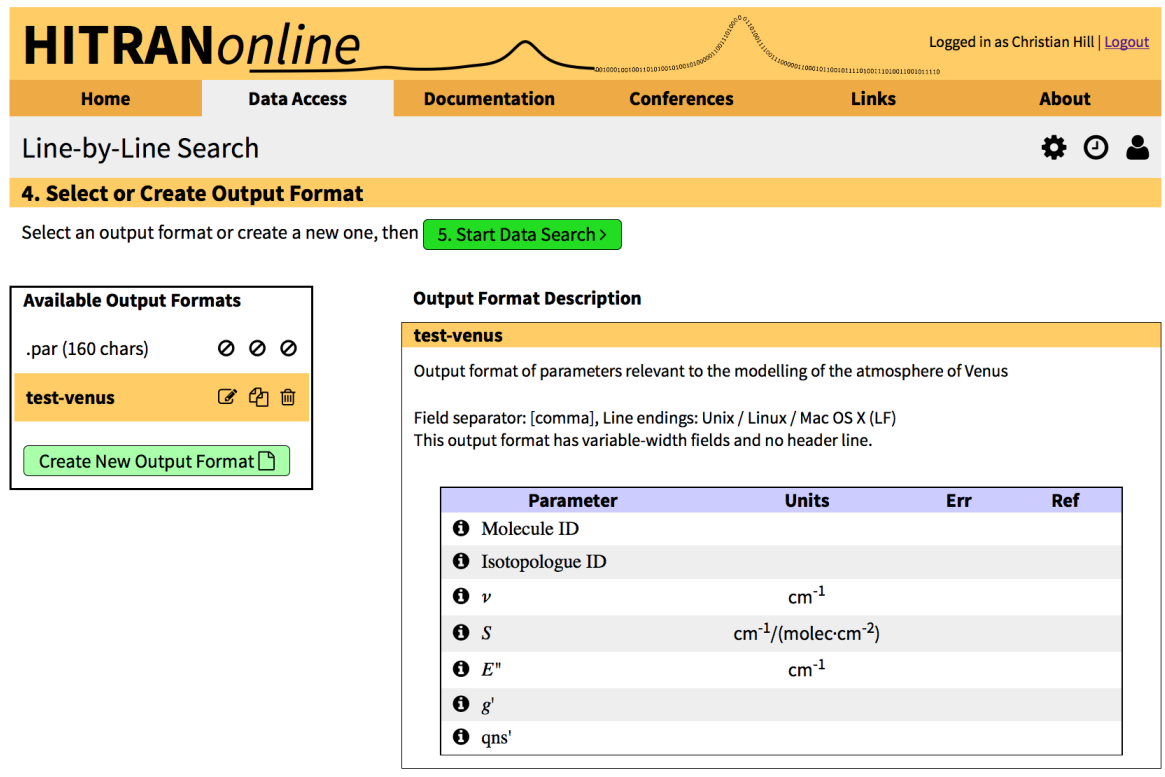

Figure 4: Step four for making a query of line-by-line data using the HITRANonline interface: Select Output Format. 


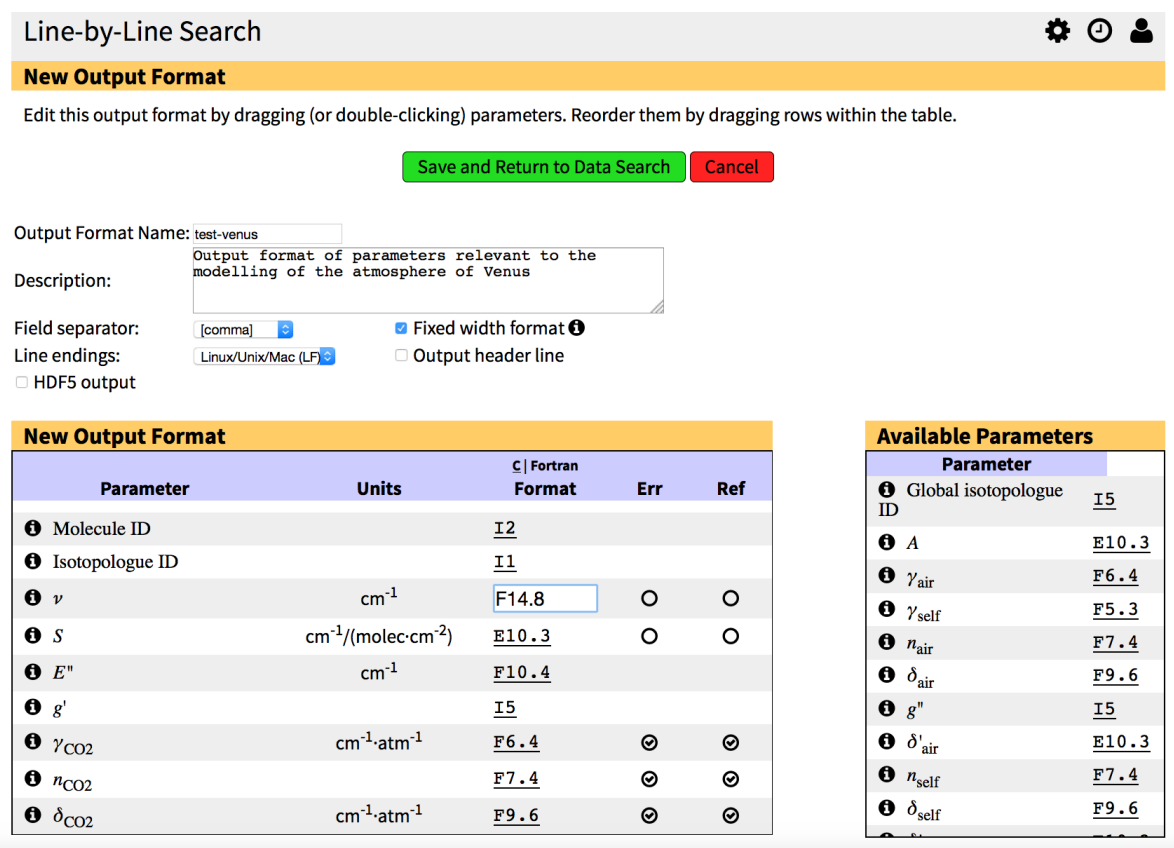

Figure 5: Editing a custom output format through the HITRANonline interface. In this screenshot, the format of the wavenumber field is being edited. 
Table 1: Parameters from the HITRAN2004 format in HITRAN online

\begin{tabular}{lcll}
\hline Parameter & Units & Identifier $^{1}$ & Description \\
\hline$\tilde{\nu}$ & $\mathrm{cm}^{-1}$ & $\mathrm{nu}$ & Transition wavenumber \\
$S$ & $\mathrm{~cm}^{-1} /\left(\mathrm{molec} \cdot \mathrm{cm}^{-2}\right)$ & $\mathrm{sw}$ & $\begin{array}{l}\text { Transition intensity, weighted by isotopologue abun- } \\
\text { dance }\end{array}$ \\
& $\mathrm{s}^{-1}$ & $\mathrm{a}$ & $\begin{array}{l}\text { Einstein A-coefficient } \\
\text { Air-broadened Lorentzian HWHM coefficient (for }\end{array}$ \\
$\gamma_{\text {air }}$ & $\mathrm{cm}^{-1} \mathrm{~atm}^{-1}$ & gamma_air & $\begin{array}{l}\text { Voigt lineshape) } \\
\end{array}$ \\
$\gamma_{\text {self }}$ & $\mathrm{cm}^{-1} \mathrm{~atm}^{-1}$ & gamma_self & Self-broadened Lorentzian HWHM coefficient (for \\
& & & Voigt lineshape) \\
$E^{\prime \prime}$ & $\mathrm{cm}^{-1}$ & elower & Lower state energy \\
$n_{\text {air }}$ & - & n_air & Temperature-dependence exponent for $\gamma_{\text {air }}$ \\
$\delta_{\text {air }}$ & $\mathrm{cm}^{-1} \mathrm{~atm}^{-1}$ & delta_air & Air pressure-induced line shift \\
$g^{\prime}$ & - & gp & Lower state statistical weight \\
$g^{\prime \prime}$ & - & gpp & Upper state statistical weight \\
\hline
\end{tabular}

\footnotetext{
${ }^{1}$ The "identifier" in this column is a plain-text (ASCII) representation of the parameter name used internally and in the
} optional header line of output data. 
Table 2: Metadata and other special "parameters"

\begin{tabular}{|c|c|c|}
\hline Parameter & Identifier & Description \\
\hline Transition ID & trans_id & Unique integer identifying the transition \\
\hline Molecule ID & molec_id & HITRAN integer identifying the molecule \\
\hline Isotopologue ID & local_iso_id & $\begin{array}{l}\text { HITRAN integer identifying the isotopologue of a par- } \\
\text { ticular molecule }\end{array}$ \\
\hline Global Isotopologue ID & global_iso_id & $\begin{array}{l}\text { Global integer ID identifying the isotopologue (unique } \\
\text { across the whole database) }\end{array}$ \\
\hline qns ${ }^{\prime}$ & statep & Upper state quantum numbers \\
\hline qns & statepp & Lower state quantum numbers \\
\hline .par line & par_line & $\begin{array}{l}\text { Complete representation of the line in the HI- } \\
\text { TRAN2004 160-character, .par format }\end{array}$ \\
\hline
\end{tabular}


Table 3: Parameters for broadening by new perturbing species, $\mathrm{X}$, and non-Voigt parameters

\begin{tabular}{|c|c|c|c|}
\hline Parameter & Units & Identifier & Description \\
\hline$n_{\text {self }}$ & - & n_self & Temperature-dependence exponent for $\gamma_{\text {self }}$ \\
\hline$\delta_{\text {self }}$ & $\mathrm{cm}^{-1} \mathrm{~atm}^{-1}$ & delta_self & Self-induced pressure line shift \\
\hline$\delta_{\text {air }}^{\prime}$ & $\mathrm{cm}^{-1} \mathrm{~atm}^{-1} \mathrm{~K}^{-1}$ & deltap_air & (Linear) temperature dependence coefficient for $\delta_{\text {air }}$ \\
\hline$\delta_{\text {self }}^{\prime}$ & $\mathrm{cm}^{-1} \mathrm{~atm}^{-1} \mathrm{~K}^{-1}$ & deltap_self & (Linear) temperature dependence coefficient for $\delta_{\text {self }}$ \\
\hline$\gamma_{\mathrm{X}}$ & $\mathrm{cm}^{-1} \mathrm{~atm}^{-1}$ & gamma_X & $\begin{array}{l}\text { Lorentzian HWHM coefficient (for Voigt lineshape) } \\
\text { for broadening by perturber X }\end{array}$ \\
\hline$n_{\mathrm{X}}$ & - & $\mathrm{n} \_\mathrm{X}$ & Temperature-dependence exponent for $\gamma_{\mathrm{X}}$ \\
\hline & $\mathrm{cm}^{-1} \mathrm{~atm}^{-1}$ & delta_X & Pressure-induced line shift due to perturber X \\
\hline$\gamma_{0}^{\mathrm{HT}}\left(X ; T_{\text {ref }}\right)$ & $\mathrm{cm}^{-1} \mathrm{~atm}^{-1}$ & gamma_HT_O_X_T & $\begin{array}{l}\text { Speed-averaged HTP half-width }{ }^{1} \text { in temperature } \\
\text { range around } T_{\text {ref }} \text { due to perturber } X\end{array}$ \\
\hline$n^{\mathrm{HT}}\left(X ; T_{\text {ref }}\right)$ & - & $\mathrm{n} \_\mathrm{HT} \_\mathrm{X} \_\mathrm{T}$ & $\begin{array}{l}\text { Temperature dependence exponent around } T_{\text {ref }} \text { for } \\
\gamma_{0}^{\mathrm{HT}}\left(X ; T_{\text {ref }}\right)\end{array}$ \\
\hline$\gamma_{2}^{\mathrm{HT}}\left(X ; T_{\mathrm{ref}}\right)$ & $\mathrm{cm}^{-1} \mathrm{~atm}^{-1}$ & gamma_HT_2_X_T & $\begin{array}{l}\text { Speed-dependence of the HTP half-width in temper- } \\
\text { ature range around } T_{\text {ref }} \text { due to perturber } X\end{array}$ \\
\hline
\end{tabular}

${ }^{1}$ For a more detailed description of the parameterization of the Hartmann-Tran profile, see [Wcisło et al. (2015)]. 
Table 3: continued

\begin{tabular}{|c|c|c|c|}
\hline Parameter & Units & Identifier & Description \\
\hline$\delta_{0}^{\mathrm{HT}}\left(X ; T_{\mathrm{ref}}\right)$ & $\mathrm{cm}^{-1} \mathrm{~atm}^{-1}$ & delta_HT_O_X_T & $\begin{array}{l}\text { Speed-averaged line shift of the HTP in temperature } \\
\text { range around } T_{\text {ref }} \text { due to perturber } X\end{array}$ \\
\hline$\delta^{\prime} \mathrm{HT}\left(X ; T_{\text {ref }}\right)$ & $\mathrm{cm}^{-1} \mathrm{~atm}^{-1} \mathrm{~K}^{-1}$ & deltap_HT_0_X_T & $\begin{array}{l}\text { Linear temperature-dependence coefficient for } \\
\delta_{0}^{\mathrm{HT}}\left(X ; T_{\text {ref }}\right)\end{array}$ \\
\hline$\delta_{2}^{\mathrm{HT}}\left(X ; T_{\mathrm{ref}}\right)$ & $\mathrm{cm}^{-1} \mathrm{~atm}^{-1}$ & delta_HT_2_X_T & $\begin{array}{l}\text { Speed-dependence of the HTP line shift in tempera- } \\
\text { ture range around } T_{\text {ref }} \text { due to perturber } X\end{array}$ \\
\hline$\beta^{\mathrm{HT}}(X)$ & $\mathrm{s}^{-1}$ & beta_HT_X & $\begin{array}{l}\text { Frequency of velocity changing collisions in the HTP } \\
\text { formulism }\end{array}$ \\
\hline$\kappa^{\mathrm{HT}}(X)$ & - & kappa_HT_X & Temperature dependence of $\beta^{\mathrm{HT}}$ \\
\hline
\end{tabular}




\subsection{Query results}

The results returned by a query are presented with a set of files for download. In addition to the data in the requested output format, is a README file describing the format and two bibliography files giving sources and notes relating to the returned data. One is an HTML file with links to the cited articles at their journal websites, the other is a .bib file containing these references in BibTeX format for inclusion in a LaTeX document.

For the example search presented in the preceding figures, the output file generated has the following format (the header line has been added for clarity):

molec_id, local_iso_id, nu, sw, elower, gp, gamma_CO2, n_CO2, delta_co2

$15,2,2600.92728300,1.700 e-70,24121.2091,648,0.0377,0.7500,-0.019000$

$15,2,2601.38953300,5.172 e-83,30906.5407,648,0.0333,0.7500,-0.027950$

$15,1,2601.94927200,4.600 e-70,24145.4057,648,0.0382,0.7500,-0.020000$

$15,2,2602.34730000,3.194 \mathrm{e}-26,3609.9069,120,0.0477,0.7500,-0.010500$

$15,1,2604.02213100,9.864 \mathrm{e}-26,3613.0846,120,0.0472,0.7500,-0.010700$

$15,2,2609.71384800,6.585 e-43,11576.7861,152,0.0448,0.7500,-0.017500$

...

If fewer than 100000 transitions are returned by the query, an interactive graph of their intensities as a function of wavenumber is also presented to the user. This graph can be panned, zoomed, and saved as an image.

If fewer than 1000 transitions are returned, they are listed in a table beneath the graph. Hovering the mouse cursor over each parameter brings up a reference for that parameter containing a link to the journal article in which it was originally published, as shown in Figure 6.

Each individual reference (journal article, note, private communication, etc.) has a unique "global" identifying integer ID which is referred to in the data file; for the convenience of users of the .par format, the "per-molecule" identifying integers are retained and cross-referenced with their global equivalents when this output format is selected.

\subsection{Searching by vibrational band}

For advanced users, there is an additional functionality that can be enabled in the user settings (accessed by clicking the "cog" icon on any page or at http://hitran.org/settings/) to allow selection of lines by vibrational band. The search steps are followed as above, but if only a single isotopologue is selected, the additional option " $3 \mathrm{~b}$. Select vibrational bands" becomes available at Step 3. Clicking this button brings up a table of all of 


\begin{tabular}{|c|c|c|c|c|c|c|c|c|c|c|}
\hline \multicolumn{2}{|c|}{ Isotopologue $v$} & $S$ & $\boldsymbol{A}$ & \multirow{2}{*}{$\begin{array}{l}\gamma_{\text {air }} \\
0.0085\end{array}$} & \multirow{2}{*}{$\begin{array}{c}\gamma_{\text {self }} \\
0.06\end{array}$} & \multirow{2}{*}{$\begin{array}{l}\boldsymbol{E}^{\prime \prime} \\
24121.2091\end{array}$} & \multirow{2}{*}{$\begin{array}{r}\boldsymbol{n}_{\text {air }} \\
0.5\end{array}$} & \multirow{2}{*}{$\begin{array}{l}\delta_{\text {air }} \\
-0.0111\end{array}$} & \multirow{2}{*}{$\begin{array}{l}J^{\prime} \\
40\end{array}$} & \multirow{2}{*}{$\begin{array}{l}J^{\prime \prime} \\
39\end{array}$} \\
\hline $\mathrm{H}^{37} \mathrm{Cl}$ & 2600.9273 & $1.7 e-70$ & 0.7388 & & & & & & & \\
\hline $\mathrm{H}^{37} \mathrm{Cl}$ & 2601.3895 & $5.172 \mathrm{e}-83$ & 47.38 & 0.0093 & 0.06 & 30906.5407 & 0.5 & -0.011 & 40 & 41 \\
\hline $\mathrm{H}^{35} \mathrm{Cl}$ & 2601.949 & $4.6 e-70$ & 0.7183 & 0.0085 & 0.06 & 24145.4057 & 0.5 & -0.0111 & 40 & 39 \\
\hline $\mathrm{H}^{37} \mathrm{Cl}$ & 2602.3473 & $3.194 \mathrm{e}-26$ & 37.64 & 0.0264 & 0.126 & 3609.9069 & 0.13 & -0.0077 & 7 & 8 \\
\hline $\mathrm{H}^{35} \mathrm{Cl}$ & 2604.0221 & $9.864 \mathrm{e}-26$ & 37.75 & 0.0264 & 0.126 & 3613.0846 & 0.13 & -0.0077 & 7 & 8 \\
\hline $\mathrm{H}^{37} \mathrm{Cl}$ & $26 n 97128$ & $65850-42$ & $\Delta \cap 52$ & $\cap \cap 199$ & $\cap 1 \cap 8$ & 115757861 & $n_{2}$ & $-\cap n 119$ & $a$ & 2 \\
\hline $\begin{array}{l}\mathrm{H}^{37} \mathrm{Cl} \\
\mathrm{H}^{35} \mathrm{Cl} \\
\mathrm{H}^{35} \mathrm{Cl}\end{array}$ & \multicolumn{10}{|c|}{$\begin{array}{l}\text { 608. G. Li, I.E. Gordon, P.F. Bernath, L.S. Rothman, "Direct fit of experimental } \\
\text { ro-vibrational intensities to the dipole moment function: Application to HCl", Journal of } \\
\text { Quantitative Spectroscopy and Radiative Transfer 112, 1543-1550 (2011). [ADS] }\end{array}$} \\
\hline $\mathrm{H}^{35} \mathrm{Cl}$ & $\angle 01 \angle .500$ & $3.151 e^{-50}$ & 59.14 & 0.0195 & 0.250 & 0305.0445 & 0.10 & -0.0054 & $<$ & $\perp$ \\
\hline $\mathrm{H}^{37} \mathrm{Cl}$ & 2612.6929 & $1.294 \mathrm{e}-81$ & 60.85 & 0.0093 & 0.06 & 30248.4994 & 0.5 & -0.011 & 32 & 33 \\
\hline $\mathrm{H}^{37} \mathrm{Cl}$ & 2615.1404 & $5.551 e-69$ & 1.315 & 0.0085 & 0.06 & 23515.2882 & 0.5 & -0.0112 & 39 & 38 \\
\hline $\mathrm{H}^{35} \mathrm{Cl}$ & 2615.19 & $2.365 e-80$ & 61.63 & 0.0096 & 0.06 & 29748.4602 & 0.5 & -0.011 & 16 & 17 \\
\hline $\mathrm{H}^{35} \mathrm{Cl}$ & 2615.90 & $1.214 \mathrm{e}-81$ & 55.21 & 0.0093 & 0.06 & 30499.9682 & 0.5 & -0.011 & 36 & 37 \\
\hline $\mathrm{H}^{35} \mathrm{Cl}$ & 2616.221 & $1.52 \mathrm{e}-68$ & 1.289 & 0.0085 & 0.06 & 23538.81 & 0.5 & -0.0112 & 39 & 38 \\
\hline $\mathrm{H}^{37} \mathrm{Cl}$ & 2616.3387 & $3.966 e-31$ & 54.47 & 0.0724 & 0.253 & 5781.6966 & 0.71 & -0.0054 & 2 & 3 \\
\hline $\mathrm{H}^{37} \mathrm{Cl}$ & 2616.4893 & $8.185 e-81$ & 61.74 & 0.0096 & 0.06 & 29732.0275 & 0.5 & -0.011 & 16 & 17 \\
\hline $\mathrm{H}^{37} \mathrm{Cl}$ & 2617.3549 & $4.339 \mathrm{e}-82$ & 55.16 & 0.0093 & 0.06 & 30476.3035 & 0.5 & -0.011 & 36 & 37 \\
\hline $\mathrm{H}^{35} \mathrm{Cl}$ & 2618.0434 & $1.218 \mathrm{e}-30$ & 54.61 & 0.0724 & 0.253 & 5785.924 & 0.71 & -0.0054 & 2 & 3 \\
\hline $\mathrm{H}^{35} \mathrm{Cl}$ & 2618.77 & $3.455 e-88$ & 1.552 & 0.0093 & 0.06 & 32821.7159 & 0.5 & -0.011 & 29 & 28 \\
\hline $\mathrm{H}^{35} \mathrm{Cl}$ & 2619.04 & $2.052 \mathrm{e}-88$ & 27.36 & 0.0093 & 0.06 & 33489.1916 & 0.5 & -0.011 & 25 & 26 \\
\hline $\mathrm{H}^{37} \mathrm{Cl}$ & 2621.6680 & $3.17 \mathrm{e}-43$ & 39.64 & 0.0157 & 0.097 & 11741.3347 & 0.35 & -0.0117 & 10 & 9 \\
\hline $\mathrm{H}^{37} \mathrm{Cl}$ & 2621.9204 & $1.278 \mathrm{e}-88$ & 1.646 & 0.0093 & 0.06 & 32803.065 & 0.5 & -0.011 & 29 & 28 \\
\hline
\end{tabular}

Figure 6: The interactive HTML table of transitions returned by a query. Hovering the mouse cursor over a parameter brings up a citation containing a link to the published article.

the vibrational bands for the selected isotopologue, along with a line count, wavenumber range and intensity range for each band.

After selecting one or more bands, the search is constrained to the selected bands (and wavenumber interval, if previously specified).

\subsection{Search history}

Registered users can view a table of their previous searches at http:// hitran.org/profile/history/ which can be reached by clicking the clock icon on the tool bar toward the top right of every page. The search history is private and not visible to other users. This table contains fields giving the date of the search, the isotopologues searched for, wavenumber range, vibrational bands, output format used and the number of lines returned by the search. There is also a direct link to the files created: the HITRANonline service caches search results for a reasonable period of time after each query is made. There is also an option to repeat the query if these results are no longer available. 


\section{Cross section data}

The HITRAN compilation contains many infrared and ultraviolet absorption cross sections for larger molecules, at a range of pressures and temperatures relevant to the terrestrial atmosphere. This part of the database can be queried at http://hitran.org/xsc/ using the convenient link under the pull-down menu "Data Access". At this page, an individual molecule can be selected from the table on the lefthand side, which brings up a summary of the cross section data associated with it in a table on the righthand side. A molecule can also be searched for by name using the search-box above the left-hand table: a match with any known name for the molecule will bring up a summary of that molecule's cross section data in the right-hand panel: see Figure 7. Sources to the data can be inspected by hovering the mouse cursor over any of the rows in this table. After selecting one or more data sets, clicking "2. Get Data" presents a page with links to the data files and a bibliography. This results page also contains an interactive graph of the cross sections which can be panned, resized, zoomed and saved. This is illustrated in Figure 8.

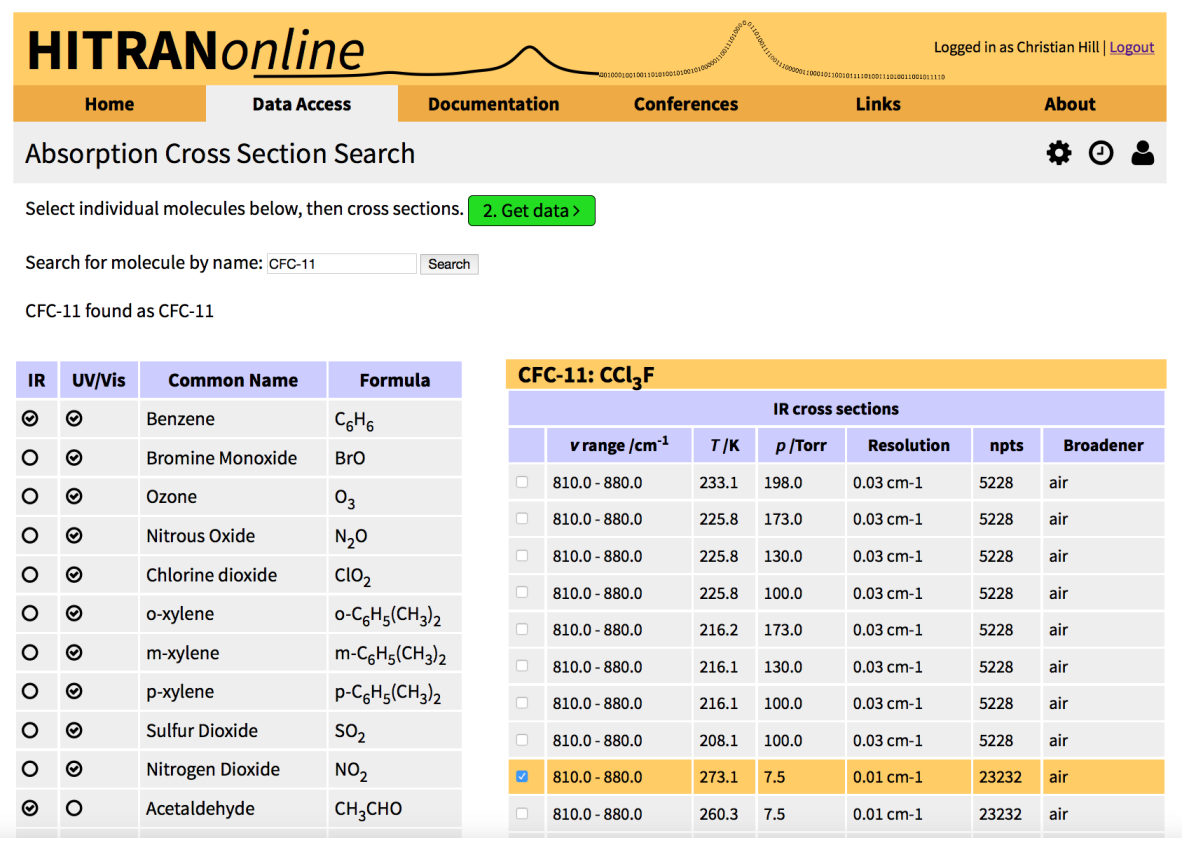

Figure 7: An absorption cross section query. 


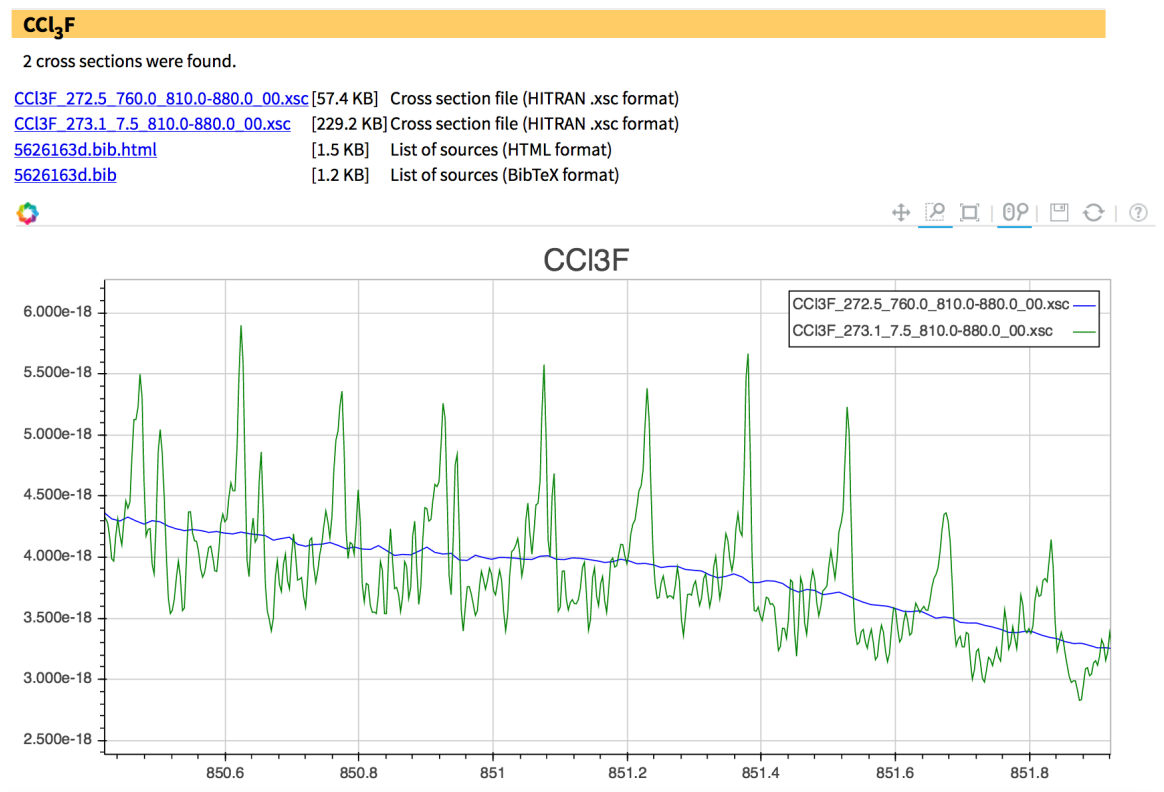

Figure 8: A plot of the cross sections at two different pressure/temperature conditions for the same molecule. The graph has been zoomed in on a narrow region of the spectrum using one of the tools provided above the plot.

\section{Other resources}

In addition to the services provided through the "Data Access" part of the HITRANonline interface, the website contains documentation, a summary of definitions and the units used in the data, news and updates, and a page of "frequently asked questions" (FAQs). The molecules and isotopologues in the database are listed with the ID numbers assigned to them and some additional information.

Of particular importance is the page of isotopologue "metadata" (available from the "Documentation" pull-down menu. This gives a table of isotopologues for each molecule, listing their natural (terrestrial) abundance (the values used in scaling the line intensities), the molar mass (necessary for calculating the Doppler half-width), and the state-independent statistical weight due to nuclear spin, $g_{i}$ [Šimečková et al. (2006)]. The value of the partition sum at the HITRAN reference temperature, $Q(296 \mathrm{~K})$, is also provided. This page contains a link to the same data in a machine-readable text file, molparam.txt, in the same format as the file with the same name that was distributed through the HITRAN website previously. 
The isotopologue metadata page also contains links to the full partition sums, $Q(T)$, for each isotopologue between 70 and $3000 \mathrm{~K}$ in $1 \mathrm{~K}$ intervals, where this has been calculated. The Total Internal Partition Sums (TIPS) code TIPS_2011.for [Laraia et al. (2011)] has been used to evaluate $Q(T)$.

Finally, the HITRAN Application Programming Interface (HAPI) library and its documentation can be downloaded from http://hitran.org/hapi/ (a link also available from the "Data Access" pull-down menu on every page). This suite of Python routines provides methods for remotely-accessing and querying the HITRANonline from within code and contains sophisticated functions for calculating spectra at different pressures, temperatures and compositions using several types of line shape and, optionally, accounting for instrument functions. Spectra can be calculated as absorption coefficients (cross sections) or transmission, absorption or radiance spectra. See [Kochanov et al. (2015)] for full details of HAPI.

\section{Conclusion and future work}

This article has described the HITRANonline website, which is the official point-of-access to the HITRAN database. The principal benefits of this new approach to obtaining HITRAN data are as follows.

- The ability to represent any number of transition parameters, including new broadening species and non-Voigt line shapes;

- each numerical parameter can be represented with up to 15 significant digits of precision with its own reference and uncertainty;

- the automated generation of a bibliography of the original data sources for each data set returned by a search;

- the ability to produce user-defined output formats, customized according to the user's needs, with control over the output precision and widths, separator and ordering of fields, and the automated generation of "README" documentation;

- interactive graphs of the selected data to aid visualization;

- a private search history for each user for recalling and repeating previous queries. 
As the HITRANonline interface is further developed, we hope to build additional functionality as follows.

- Collision-induced absorption: currently, files relating to collision-induced absorption cross sections are provided as downloads from the HITRANonline website. We hope to provide these through a searchable interface similar to that used for absorption cross sections in future.

- Line-mixing: for the absorption bands of some molecules, line-mixing effects must be taken into account for an accurate description of the spectrum. We intend to extend the existing data model to provide the parameters necessary to represent this effect through the HITRAN online interface.

- Very large data sets, such as the line-by-line transitions lists for $\mathrm{SF}_{6}$, $\mathrm{ClONO}_{2}$ and $\mathrm{CF}_{4}$, and the high-temperature compilation HITEMP [Rothman et al. (2010)] (which contains over 120 million lines) are not currently represented in the relational database backend that underlies HITRANonline and these data are currently available only as downloadable text files. A simple search functionality (probably only by wavenumber range and species) will be provided for these data collections in future.

- Units. For historical reasons and by widespread convention in the radiative transfer community, HITRAN has mostly used cgs units in its data compilations. We hope to provide an option within HITRANonline for the conversion of data sets to user-defined units systems, chosen through the user's profile settings.

The HITRANonline maintainers welcome feedback from users to continue to improve the interface: such feedback can be given by contacting the HITRAN group through the links given on the website, and at the email address info@hitran.org.

\section{Acknowledgments}

Support from NASA Planetary Atmospheres Grant NNX13AI59G and the NASA AURA program Grant NNX14AI55G is gratefully acknowledged. 
LB was sponsored by the Smithsonian Astrophysical Observatory Latino Initiative Program. This program received Federal support from the Latino Initiatives Pool, administered by the Smithsonian Latino Center. We would also like to thank P. Wcisło and the HITRAN committee for testing, validating and providing feedback on the HITRANonline service.

We are very thankful to Yu. L. Babikov, S. N. Mikhailenko and S. A. Tashkun for their work on "HITRAN on the Web", which suggested some of the interface functionalities that were implemented in HITRANonline.

\section{Appendix A. Hardware and software stack}

The HITRANonline web application runs on a Virtual Machine cloud server provisioned with 8 cores and 14 GB of RAM. The operating system is Ubuntu 14.04.3 LTS (kernel version Linux version 3.13.0-65-generic). The software stack is as follows:

- Apache 2.4.7

- Python 2.7

- Django 1.8

- MySQL 5.5

The website user interface is written in HTML/CSS and JavaScript/jQuery.

\section{References}

Boone, C. D., Walker, K. A., Bernath, P. F., 2007. Speed-dependent Voigt profile for water vapor in infrared remote sensing applications. Journal of Quantitative Spectroscopy and Radiative Transfer 105, 525-532.

Charbonneau, D., Brown, T. M., Noyes, R. W., Gilliland, R. L., Mar. 2002. Detection of an Extrasolar Planet Atmosphere. The Astrophysical Journal $568,377-384$.

Galatry, L., 1961. Simultaneous Effect of Doppler and Foreign Gas Broadening on Spectral Lines. Physical Review 122, 1218-1223. 
Hartmann, J.-M., Boulet, C., Robert, D., 2008. Collisional effects on molecular spectra. Laboratory experiments and models, consequences for applications. Elsevier, Amsterdam.

Hill, C., Gordon, I. E., Rothman, L. S., Tennyson, J., Nov. 2013. A new relational database structure and online interface for the HITRAN database. Journal of Quantitative Spectroscopy and Radiative Transfer 130, 51-61.

Jaffe, L. D., Herrell, L. M., 1997. Cassini/Huygens Science Instruments, Spacecraft, and Mission. Journal of Spacecraft and Rockets 34, 509-521.

Kochanov, R. V., Gordon, I. E., Rothman, L. S., Wcisło, P., Hill, C., Wilzewski, J. S., 2015. HITRAN Application Programming Interface (HAPI): a comprehensive approach to working with spectroscopic data. Journal of Quantitative Spectroscopy and Radiative Transfer, submitted.

Laraia, A. L., Gamache, R. R., Lamouroux, J., Gordon, I. E., Rothman, L. S., 2011. Total internal partition sums to support planetary remote sensing. Icarus 215 (1), 391-400.

Ngo, N. H., Lisak, D., Tran, H., Hartmann, J. M., 2013. An isolated lineshape model to go beyond the Voigt profile in spectroscopic databases and radiative transfer codes. Journal of Quantitative Spectroscopy and Radiative Transfer 129, 89-100.

Ngo, N. H., Lisak, D., Tran, H., Hartmann, J. M., Feb. 2014. Erratum to "An isolated line-shape model to go beyond the Voigt profile in spectroscopic databases and radiative transfer codes". Journal of Quantitative Spectroscopy and Radiative Transfer 134, 105.

Rautian, S. C., Sobel'Man, I. I., 1966. The Effect of Collisions on the Doppler Broadening of Spectral Lines . Usp. Fiz. Nauk. 90, 209-236.

Rothman, L., Rinsland, C., Goldman, A., Massie, S., Edwards, D., Flaud, J. M., Perrin, A., Camy-Peyret, C., Dana, V., Mandin, J. Y., Schroeder, J., McCan, A., Gamache, R., Wattson, R., Yoshino, K., Chance, K., Jucks, K., Brown, L., Nemtchinov, V., Varanasi, P., 1998. The HITRAN Molecular Spectroscopic Database and HAWKS (HITRAN Atmospheric Workstation): 1996 Edition. Journal of Quantitative Spectroscopy and Radiative Transfer 60, 665-710. 
Rothman, L. S., Gordon, I. E., Babikov, Y., Barbe, A., Benner, D. C., Bernath, P. F., Birk, M., Bizzocchi, L., Boudon, V., Brown, L. R., Campargue, A., Chance, K., Cohen, E. A., Coudert, L. H., Devi, V. M., Drouin, B. J., Fayt, A., Flaud, J.-M., Gamache, R. R., Harrison, J. J., Hartmann, J.-M., Hill, C., Hodges, J. T., Jacquemart, D., Jolly, A., Lamouroux, J., LeRoy, R. J., Li, G., Long, D. A., Lyulin, O., Mackie, C., Massie, S. T., Mikhailenko, S., Müller, H. S., Naumenko, O., Nikitin, A., Orphal, J., Perevalov, V. I., Perrin, A., Polovtseva, E. R., Richard, C., Smith, M. A. H., Starikova, E., Sung, K., Tashkun, S., Tennyson, J., Toon, G. C., Tyuterev, V. G., Wagner, G., 2013. The HITRAN 2012 Molecular Spectroscopic Database. Journal of Quantitative Spectroscopy and Radiative Transfer 130, 4-50.

Rothman, L. S., Gordon, I. E., Barber, R. J., Dothe, H., Gamache, R. R., Goldman, A., Perevalov, V. I., Tashkun, S. A., Tennyson, J., 2010. HITEMP, the high-temperature molecular spectroscopic database. Journal of Quantitative Spectroscopy and Radiative Transfer 111 (15), 2139-2150.

Rothman, L. S., Jacquemart, D., Barbe, A., Benner, D. C., Birk, M., Brown, L. R., Carleer, M. R., Jr., C. C., Chance, K., Coudert, L. H., Dana, V., Devi, V. M., Flaud, J.-M., Gamache, R., Goldman, A., Hartmann, J.-M., Jucks, K., Maki, A., Mandin, J.-Y., Massie, S. T., Orphal, J., Perrin, A., Rinsland, C. P., Smith, M. A. H., Tennyson, J., Tolchenov, R. N., Toth, R. A., Auwera, J. V., Varanasi, P., Wagner, G., 2005. The HITRAN 2004 molecular spectroscopic database. Journal of Quantitative Spectroscopy and Radiative Transfer 96, 139-204.

Šimečková, M., Jacquemart, D., Rothman, L., Gamache, R., Goldman, A., 2006. Einstein $A$-coefficients and statistical weights for molecular absorption transitions in the HITRAN database. Journal of Quantitative Spectroscopy and Radiative Transfer 98, 130-155.

Wcisło, P., Gordon, I. E., Tran, H., Tan, Y., Hu, S.-M., Campargue, A., Kassi, S., Romanini, D., Hill, C., Kochanov, R. V., Rothman, L. S., 2015. The implementation of non-Voigt line profiles in the HITRAN database: $\mathrm{H}_{2}$ case study. Journal of Quantitative Spectroscopy and Radiative Transfer, submitted.

Wilzewski, J. S., Gordon, I. E., Kochanov, R. V., Hill, C., Rothman, L. S., 2016. $\mathrm{H}_{2}, \mathrm{He}$, and $\mathrm{CO}_{2}$ line-broadening coefficients, pressure shifts and 
temperature-dependence exponents of $\mathrm{SO}_{2}, \mathrm{NH}_{3}, \mathrm{HF}, \mathrm{HCl}$, OCS and $\mathrm{C}_{2} \mathrm{H}_{2}$ for the HITRAN database. Journal of Quantitative Spectroscopy and Radiative Transfer 168, 193-206. 